\title{
A method for investigation of spinal kinematics in children with idiopathic scoliosis
}

\author{
Paweł Maciejasz*1, Wiesław Chwała ${ }^{2}$, Marianna Białek ${ }^{3}$ and \\ Tomasz Kotwicki ${ }^{4}$
}

Address: ${ }^{1}$ Institute of Precision and Biomedical Engineering, Warsaw University of Technology, Warsaw, Poland, ${ }^{2}$ Department of Biomechanics, Laboratory of Biokinetics, Academy of Physical Education, Cracow, Poland, ${ }^{3}$ Fits Center Kielce, Poland and ${ }^{4}$ Department of Pediatric Orthopedics and Traumatology, University of Medical Sciences, Poznan, Poland

* Corresponding author

from $5^{\text {th }}$ International Conference on Conservative Management of Spinal Deformities

Athens, Greece. 3-5 April 2008

Published: 15 January 2009

Scoliosis 2009, 4(SuppI I):O2I doi:I0.II86/I748-7|6I-4-SI-O2I

This abstract is available from: http://www.scoliosisjournal.com/content/4/SI/O2I

(C) 2009 Maciejasz et al; licensee BioMed Central Ltd.

\section{Background}

The present motion capture systems utilizing surface markers are not ideal. It is difficult to calculate the location of the particular vertebrae. To better study scoliosis, a noninvasive, tool is needed which can study spine kinematics during exercise.

\section{Purpose}

To develop and to evaluate the reliability of a novel algorithm (model). The goal is to utilize this algorithm while investigating the kinematics of idiopathic scoliosis with use of a motion capture system.

\section{Methods}

The developed model uses surface markers to determine positions and orientations of the head, C7 vertebra and the sacrum. Next, Bézier curves and the radiological data (scoliosis size and location) are configured to connect the segments (head, C7 and sacrum) and to create a model of the whole spine.

Dynamic trials were performed in order to verify the algorithm. The spine kinematics of 8 juveniles suffering from idiopathic scoliosis were investigated during gait, lateral bending and twisting. Additional markers were placed on the skin over a few vertebrae in order to compare their physical locations with calculated locations.

\section{Results}

High repeatability of peak scoliosis angles and moments of its occurrences during gait cycles were observed.

Even when performing complicated tasks (lateral bending or twisting), calculated position of vertebrae where in good agreement with surface markers indicating their localization.

\section{Conclusion}

The developed model has a potential to evaluate complex kinematics of a spine. However, to introduce this method in scoliosis management further improvements and investigations are necessary.

\section{References}

I. Allard P, Stokes IAF, Blanchi JP: Three - dimensional Analysis of Human Movement. Human Kinetics, New York; 1995.

2. Labelle H, Dansereau J, Bellefleur C, Jéquier JC: Variability of geometric measurements from three-dimensional reconstructions of scoliotic spines and rib cages. European Spine Journal 1995, 4(2):88-94.

3. Moeslund TB, Hilton $A, K$ Küger $V$ : $A$ survey of advances in visionbased human motion capture and analysis. Computer Vision and Image Understanding 2006, 104(2):90-126. 